\title{
Study on Mode of Brand Communication Based on Micro-message Public
}

\author{
Platform
}

\author{
Julan Zhang \\ No.449 Beijing Road School of Journalism and communication of Xinjiang University Of Finance \\ and Economics, Urumqi, Xinjiang,China \\ 57385969@QQ.com
}

\begin{abstract}
:
Micro-message marketing propagation has been widely recognized and accepted by most people, however, only a few domestic enterprises can successfully use micro-message as a means of marketing communication to conduct effective promotion of the brand, and most of the enterprises still can't find brand marketing communication strategy based on micro message public platform(MMPP for short).In this paper, with the aid of communication theory and marketing theory, further makes an in-depth study on the media brand communication modes of MMPP which is different from traditional medias and other network medias based on the analysis of the advantage of brand's marketing communication in public platforms represented by self-media .
\end{abstract}

Keywords: Micro-message; Brand Communication; Marketing Strategies

\section{Theoretical Basis of MMPP Brand Communication}

Micro-message, also known as We-Chat, is a phone chatting software developed by Tencent in 2011 for users to send written message, photos and intercom voice. Users can send voice message, video message, written message and photos rapidly through phone or tablet personal computer, meanwhile, they can also enjoy the services such as "shake it off", "circle of friends", "public platform" and "rich scan".

Micro-message various obstacles in operators, hardware and software, and social networking services (SNC for short), which has realizes the seamless joint between the real and the virtual world, enabling the mobile terminal to become a new social node. Effective brand communication based on the network self-media has become a hot issue concerned by brand holders. There are many principles of communication in the brand communication of self-media, so, a better understanding of these principles can help brand holders to formulate relevant strategies for brand communication of MMPP.

\subsection{Symbolic Interaction Theory}

Symbolic interaction theory, hereinafter refers to as SI theory, is a kind of thinking way towards "thinking", "self" and "society". G, H, Mead is generally regarded as the founder of the theory. The three most important concepts proposed by Mead are "social", "self" and "thinking", in which he thought a person's heart belongs to society and develops through the interaction and communication with others,

Symbolic interaction theory has made a great contribution to the social and cultural traditions, which guides people to achieve the goal of self-cognition and self-realization through meaningful interactions in a social situation. Since the advent of internet blogs, micro-blog and micro message, people are convenient to spread individual symbols and receive the other individuals' attitude, so as to realize the self-cognition. At the same time, it has a great reference value for brand communication.

\subsection{Two-step flow of communication}

P.F. Lazasfeld's book The People's Choice in 1944 firstly put forward to the theory of "two-step flow of communication", whose most important contribution is to emphasize the important role of interpersonal communication and other social factors in the process of transmission. 
The two-step flow of communication theory is not only applicable to the traditional mass media, but also more applicable to the emerging Internet media. In micro message, many individuals and companies are from various industries and fields, but act as the role of opinion leaders, providing information, opinions and comments for others and influencing others, which accelerates the speed and expands the influence of micro message. Due to micro message is a kind of social tool, everyone can become a subject of opinion leaders.

\section{Advantage analysis of brand communication in MMPP}

\subsection{The unprecedented stickiness and interactivity}

The emergence of self-media weakens the mass media's influence and elite's discourse power, but brings the interactivity of rich variety and makes communication process more multivariate and interactive. Users can independently spread the content of various forms they want to express, which can be text, images, video, music and so on. User can also comment and forward mutually. Especially since the integrity between Internet and the media, communication becomes more direct and efficient. In addition, micro message also has higher stickiness than traditional media because of its low threshold, which can meet the multiple needs of users.

\subsection{Accurate segmentation of the crowd}

The appearance of micro message provides a more reliable channel for the precision marketing of brand. It is totally different from BBS, Douban and micro-blog, users can meet those who have the same hobby interest or are in the same field through self-tags. Now the appearance of micro message shows the importance of interpersonal relationships. For the brand, if an accurate user is found, this sometimes means finding a group of users. According to their attributes, states, interests and preference, the effective and precise transmission can be worked out.

\subsection{Strong word-of-mouth effect}

Most of the brands have been aware of the importance of word-of-mouth spread, so, more and more brands root in a variety of interpersonal communication groups to develop good word of mouth. Word of mouth is not the brand advertising and promotion, but consumers' establishment of individual preferences to the brand according to their own good experience, and then diffusion of the experience and preferences to their interpersonal relationships in the form of word of mouth.

Micro message is such an ideal field of word of mouth. A simple dynamic state is likely to attract the attention of new customers, and such word of mouth effect could be amplified, therefore, micro message public platform can indeed provide a broad stage to brand reputation spread.

\subsection{Low-cost brand communication}

As is known to all, advertising in the traditional mass media is very expensive, enterprise often will spend hundreds of thousands dollars on advertising, which can not necessarily play a good effect.

Since the advent of self-media age, all brands stand in the same starting line. They just need to apply for an individual account and build a better communication effect than advertising of public relations according to consumers' habits and hobbies. This low profile way of communication not only saves a large amount of advertising expenses for the brand, but also appears warm and peaceful, which is advantageous for the consumers to accept brand. Effectively in the media communication, of course, is not so simple a word, it needs a brand can very understanding their target group, and focused to do content and services and maintenance, only in the cost of the heart and can recycle considerable communication effect.

\section{MMPP based brand communication mode}

No matter how times changes and no matter how media develops, the enterprise's competitive advantage is always determined by a strong brand. Value of the brand can transmit the product and service timely and accurately to the target consumers. In the self-media era, consumers is under fresh, independent and noisy communication environment, so, a lot of people have rebellious attitude towards the brand without originality and sincerity, as a matter of course, brand communication effect is not as well as before in such context. E enterprises must change their brand communication mode and strategy in order to adapt to 
the variable and personalized self-media environment, cater to the tastes of consumers, and to prove their brand value

\subsection{Precision marketing model}

In the era of mobile internet, micro message is the pioneer and the best carrier of precise marketing. The main role of micro message in the brand communication is not dependent on the number of fans and the large number of communication to enhance the brand visibility, but based on the user's active choice, thus to promote the results-oriented marketing communication goals. In this process, the brand can realize the control of information dissemination and improve communication feedback, so as to improve the validity of information communication and accuracy of marketing communication.

\subsection{Service marketing model}

Service marketing is one of the types of marketing, which is derived from the marketing, and is a development of the marketing. In traditional relationship marketing, enterprises are usually easier to maintain relationships with suppliers, distributors and dealers. But because of the lack of effective channels, it is difficult for enterprises to directly contact with consumers. Since the advent of micro message, enterprises are able to better interact with consumers directly. Due to its nature of privacy, micro message is more suitable to be used as an effective service platform to develop deep relations between enterprises and consumers.

\subsection{Word of mouth mode}

Word of mouth's importance to the merchants is self-evident, which is usually classified into the category of brand reputation. Enterprises with high reputation will be better in their survival and development. The MMPP is provided with a great potential of precision marketing, for it can effectively improve the efficiency of network word-of-mouth brand. In the Internet word-of-mouth spread, enterprises need to accurate grasp of the circle of users and their relations according to different user activity circles, in order to know about what kind of fields and products they may be interested in, and then take the initiative to attack into the circle, satisfy the opinion leaders, cultivate their brand preference and brand loyalty, and then start their word-of-mouth Combining precision marketing and word-of-mouth communication, which can effectively improve the efficiency of brand communication

\subsection{Emotion communication model}

The core to maintain the relationship between consumers and brands is to combine the brands and the consumers' emotions, for emotion of brand is an important composition element of the brand value. Enterprises can organize marketing activities shaping consumers' emotion to the brand, so the brand emotion or attachment can raise consumer's perception of the brand value, and enhance the brand assets. The purpose of brand communication is not to let consumers directly buy the products or services, but to establish an emotional connection with consumers, then to build consumer loyalty. Only when the brand has an emotional relationship with their customers, can it truly become a brand.

\section{Conclusion}

MMPP brings together a variety of individuals, and there are also many enterprises and institutions, but in essence, it belongs to the individual user's platform, the reason why people love it mostly is that individuals with similar emotions, life experience, joys and sorrows to the ordinary users gather here. In this platform, although people also pay close attention to the brand, they are not willing to accept a top-down advertising, and more reluctant to be brainwashed by various blunt brand information. What Micro message users really pursue is a kind of equality and interactive experience. The non-individuals such as brand enterprises and institutions will build a wall in the heart of grassroots users. This wall is not necessarily something unable to break through, and the best way to break through it is the establishment of human emotion. Brand actually is not an institution with cold symbol, instead, it should also has people-oriented characteristics, placing people's emotional factors in the important position.

The advent of micro message puts forward to an epoch test for all brands, which makes these brands think about how to use micro message to market themselves 
effectively. Of course, as a new thing, the development of micro message is yet to be perfect, and it seems too early to give it any conclusion, but the brand owners should face up to the trend of times, so that they can stand in an impregnable position in a new round of brand war.

\section{References:}

[1]Dan Gillmor, We the Media [M].0'Reilly MediaJnc.2006-01

[2] G, H, Mead, Mind, Self and Society [M]. Shanghai Translation Publishing House, 2008

[3] Raran S.J, Davis D.K. Mass Communication Theory: Foundations, Ferment and Future [M]. Tsinghua University Press, 2004

[3] Caywood,C.L.,Schultz,D.E.,Wang,P. IMC: A survey of consumer good advertisers. Northwestern University Report[J].1991

[4]Shayne Bowman, Chris Willis. We Media-How audience are shaping the future of news and information [M].The Media Center, 2003.6. 